\title{
Direct Neck Lift in Massive Weight Loss Patients - Classification and Treatment Algorithm
}

\author{
Ulrich Eugen Ziegler ${ }^{1}$, Ruth Schaefer ${ }^{2}$, Adrien Daigeler ${ }^{2}$, Philip Helge Zeplin ${ }^{3, *}$ \\ ${ }^{1}$ Praxis Dr. Ziegler, Stuttgart, Germany \\ ${ }^{2}$ Department of Hand, Plastic and Reconstructive Surgery, University of Tuebingen, Tuebingen, Germany \\ ${ }^{3}$ Ludwigsburg Institute of Plastic Surgery, Schlosspark Klinik Ludwigsburg, Ludwigsburg, Germany
}

Email address:

p.zeplin@schlosspark-klinik.com (P. H. Zeplin)

${ }^{*}$ Corresponding author

\section{To cite this article:}

Ulrich Eugen Ziegler, Ruth Schaefer, Adrien Daigeler, Philip Helge Zeplin. Direct Neck Lift in Massive Weight Loss Patients Classification and Treatment Algorithm. Journal of Surgery. Vol. 9, No. 2, 2021, pp. 58-62. doi: 10.11648/j.js.20210902.13

Received: March 2, 2021; Accepted: March 13, 2021; Published: March 22, 2021

\begin{abstract}
The neck is an often-neglected part of body lift procedures after massive weight loss. The objective of this study was to classify massive weight loss patients undergoing direct neck lift procedures and provide a standardized treatment algorithm. A retrospective review of all neck lift procedures performed between January of 2012 and December of 2019 was conducted. Patients were classified as follows: Grade I, no skin excess with remaining preplatysmal fat and no platysmal laxity; Grade IIa, moderate submental skin excess with subplatysmal fat deposits and platysmal laxity; Grade IIb, moderate anterior neck skin excess with no fat deposits and platysmal laxity; Grade III, considerable anterior skin excess with no or limited fat deposits and platysmal laxity and bands. Grade I patients had Liposuction, Grade IIa patients Submental Neck Lift with anterior fat excision and central platysmaplasty, Grade IIb Submental Neck Lift with central platysmaplasty, and Grade III patients Median Z-Plasty with fat excision (if needed) and central platysmal resection and tightening. Thirty-one patients were included for analysis. One underwent liposuction, twenty-five a Submental Neck Lift and five a Median Z-Plasty. Concomitant surgery was carried out in all cases. A recurrent skin laxity occurred in $2(11.8 \%)$ patients, so in local anesthesia an operative revision was required. A standardized algorithmic approach for neck lift in massive weight loss patients may optimize the result without increasing the complication rate. Direct neck lift procedures prove to be reliable techniques with rare complication rates that can be performed together with other postbariatric procedures.
\end{abstract}

Keywords: Massive Weigh Loss, Body Contouring, Neck Lift, Z-plasty, Submental

\section{Introduction}

Redundant midline neck skin is a common cosmetic problem in older patients. The deformity known as "turkey neck" also presents in younger patients after massive weight loss (MWL). Just like other regions of the body the central neck in MWL patients often is accompanied by stretch marks, making direct excisions of damaged tissue favorable over lateral neck lift approaches. The anterior cervicoplasty or zorro-scar-plasty was introduced in the 1970s by Cronin and Biggs and mostly favored in male patients [1,2]. Due to scarring along the midline the technique has fallen out of favor compared to newer procedures with hidden scars.

MWL patients tend to be younger than patients seeking face and neck lifts for rejuvenating reasons or consider a facelift too large of procedure.

Therefore, the direct submental incision neck lift as described by Singer et al. [3] and Gryskiewicz et al. [4] with less obvious scars constitutes a good alternative for weight loss patients with good skin quality but excess submental fatty tissue $[5,6]$.

For MWL patients the neck often remains an unpleasant reminder of redundant skin after major procedures of body contouring have been performed [7-10].

Though this region is a major concern for a lot of patients due to visibility, in the staged surgeries these facial reconstructions usually form the last step of the path of body contouring [7].

In contrast to aging patients, in whom the excess skin 
usually includes the entire neck area, we often only observe excess skin in patients after MWL, which is limited to the anterior neck area.

For this reason, a classification such as that introduced by Pelle-Ceravolo et al. [11] cannot be transferred to the MWL neck without restriction.

In addition, the Pittsburgh Rating Scale for contour deformities after MWL [12] does not include the neck. We therefore propose a classification into three grades of deformities for changes in the weight loss patient's neck. Additionally we developed a treatment algorithm for neck deformities in weight loss patients.

\section{Material and Methods}

We state that all procedures conformed to the Declaration of Helsinki. This study was approved by the Ethics Committee of the appropriate authority and informed written consent to publish personal and medical information was obtained from all patients.

\subsection{Patients and Methods}

A total of thirty-one patients (29 female, 2 male) after massive weight loss get a neck lift procedure between January 2012 and December 2019 were included in this retrospective study.

Patients were included if the primary concern was the fat and neck skin laxity. They refused a standard neck and face lift and clearly understood the resulting scarring.

A retrospective chart review was performed to obtain data regarding patient age, body mass index (BMI), BMI loss, comorbidities, smoking and concomitant surgery as well as complications documented and graded by the Clavien-Dindo classification for surgical complications [13].

All operations were carried out by a single surgeon (U. Z.).

\subsection{Surgical Technique}

\subsubsection{Submental Neck Lift}

For the Submental Neck Lift markings were done with patient in slightly raised position. The midline was marked and submental fold marked as anterior incision line as described before $[14,15]$. Now with head reclined the maximal skin resection was determined and the dorsal incision line marked creating an elliptical skin resection (Figure 1).

All patients received a tumescent anesthesia. An incision was made direct under the horizontal skin fold of the chin and preplatysmal preparation extended down the central neck. The platysma was addressed, bands resected and fat pads removed followed by a midline platysma corset plication as described before [16-19] to restore the cervicomental angle. The skin was draped over the resulted neckline and excess tailor tucked tension free under the chin with an elliptical skin excision.

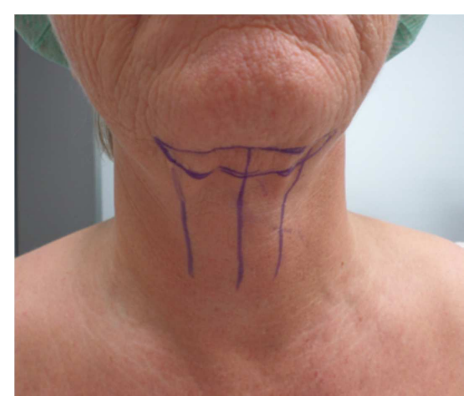

Figure 1. Submental Neck Lift: Preoperative markings of the elliptical skin resection area.

\subsubsection{Median Z-Plasty}

For the Median-Z-Plasty a vertical skin reduction was performed by a multiple z-plasty or star shape resection running down the midline of the neck. Preoperative markings with patient in upright position determined the skin redundancy by skin pinching (Figure 2).
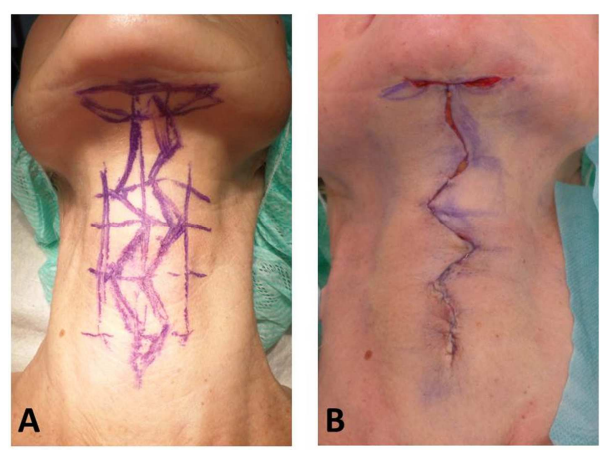

Figure 2. Median Z-Plasty: Preoperative markings (A) and postoperative findings $(B)$.

The turning points of resection lines were positioned in skin folds to prevent hypertrophic scarring or widening. After tumescent anesthesia resection was carried out according to the markings. The supra- und subplatysmal fat pads were addressed and reduced as necessary without further undermining. Platysma laxity was addressed and if needed bands resected and platysma corset plication was then performed. Skin was closed according to preoperative planning, in most cases the scar ended in an additional submental ellipse to prevent skin redundancies and dogears.

\subsubsection{Postoperative Regimen}

Patients received intraoperative taping of the scars that covered the ventral neck down to the sternal notch to prevent hematoma and swelling and redress the skin in proper position. After local tumescence anesthesia faded only systemic oral analgesia with NSAR was necessary.

Six months after surgery follow up appointment was scheduled and all patients returned for clinical evaluation and photo documentation.

\subsection{Classification}

Currently no classification for neck deformities after weight loss exists and therefore the operation planning is still 
very individual. We therefore classified our patients into four grades for weight loss induced deformities of the neck.

Patients showing no skin excess with remaining preplatysmal fat and no platysmal laxity were classified as Grade I. Those who showed moderate submental skin excess with subplatysmal fat deposits and platysmal laxity were classified as Grad IIa, same but without fat deposits were classified Grad IIb. Patients showing considerable anterior skin excess with no or limited fat deposits.

And platysmal laxity and bands were classified as Grade III (Figure 3).
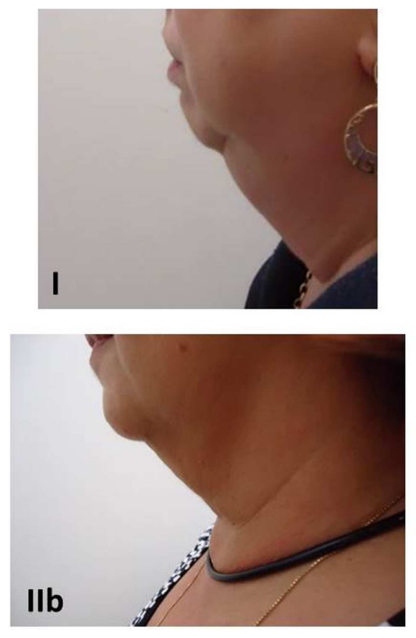
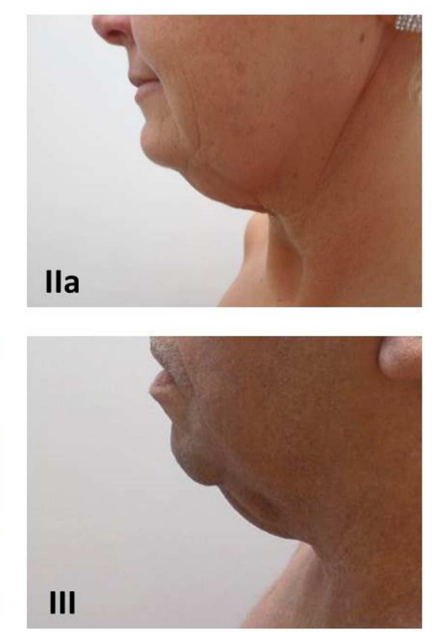

Figure 3. Grades of Neck Contour Deformities after Massive Weight Loss.

\section{Results}

Mean age of the thirty-two patients (29 female; 2 male) was $50.6 \pm 8.8$ years, the mean BMI was $29.57 \pm 4.68 \mathrm{~kg} / \mathrm{m}^{2}$. Mean BMI-loss was $17.84 \pm 8.21 \mathrm{~kg} / \mathrm{m}^{2}$ and average weight was $82 \pm 13.24 \mathrm{~kg}$.

Women were by far the predominant gender (93.5\%) only two male patients received a direct neck lift.

More than half $(67.7 \%)$ of the patients had pre-existing comorbidities, most notably smoking $(41.2 \%)$, obesity with BMI still over 30 (33.3\%) and hypertension (12.9\%). Weight loss was achieved in $58.1 \%$ of cases by bariatric surgery. Six of 31 patients $(19.4 \%)$ received gastric bypass operation, 12 (38.7\%) sleeve gastrectomy and $13(41.9 \%)$ achieved the weight loss with sports and dietary changes only.

The majority $(n=25 ; 80.7 \%)$ of patients underwent Submental Neck Lift including submental lipectomy, one $(\mathrm{n}=1 ; 3.2 \%)$ patient with a submental fat excess was treated by liposuction of the ventral neck only and five $(n=5 ; 16.1 \%)$ patients had a Median Z-Plasty.

Concomitant surgery was carried out in all $31(100.0 \%)$ cases. A recurrent skin laxity occurred in two (11.8\%) patients, so in local anesthesia an operative revision was required (Clavien-Dindo Type IIIa complication [13]).

According to our classification, this results in a treatment algorithm for the respective grade of neck contour deformity (Table 1).
Table 1. Grades of Neck Contour Deformity and Treatment Recommendation.

\begin{tabular}{|c|c|c|}
\hline Grade & Neck Contour Deformities & Treatment \\
\hline I & $\begin{array}{l}\text { No skin excess } \\
\text { Remaining preplatysmal fat } \\
\text { No platysmal laxity }\end{array}$ & Liposuction \\
\hline IIa & $\begin{array}{l}\text { Moderate submental skin excess } \\
\text { Subplatysmal fat deposits } \\
\text { Platysmal laxity }\end{array}$ & $\begin{array}{l}\text { Submental Neck Lift } \\
\text { Direct anterior fat resection } \\
\text { Central Platysma Plasty }\end{array}$ \\
\hline $\mathrm{IIb}$ & $\begin{array}{l}\text { Moderate anterior neck skin excess } \\
\text { No fat deposits } \\
\text { Platysmal laxity }\end{array}$ & $\begin{array}{l}\text { Submental Neck Lift } \\
\text { Central Platysma-Plasty }\end{array}$ \\
\hline III & $\begin{array}{l}\text { Considerable anterior skin excess } \\
\mathrm{No} / \text { limited fat deposits } \\
\text { Platysmal laxity and bands }\end{array}$ & $\begin{array}{l}\text { Median Z-Plasty } \\
\text { Fat resection (if needed) } \\
\text { Central Platysma Resection } \\
\text { and tightening }\end{array}$ \\
\hline
\end{tabular}

\section{Discussion}

Current classifications for neck laxity are for aging but not for massive weight loss patients.

So they are not applicable for the planning of operations because MWL patients tend to be younger than patients seeking face and neck lifts for rejuvenating reasons or consider a facelift too large of procedure.

In our study just like in most previous reports [20-22] women account for almost $90 \%$ of face and neck lift patients.

In weight loss patients no additional liposuction of the neck needed to be performed before direct neck lift.

This hints to the fact that weight loss effectively depletes of the superficial fat pad of the neck [23, 24].

Despite the high number of smokers $(41.2 \%)$ we did not encounter any wound dehiscence, wound margin necrosis or delayed wound healing.

Also no cases of hematoma or seroma were found. Smokers and former smokers were informed that they are prone to higher rates of wound complication [25-27], which might have led them to choose this less invasive procedure. Also the minimal need to undermine skin and the tension free wound adaption of this technique after platysmal plication is an essential reason for minimal wound complications and further delicate scarring.

During the follow up period scarring became less visible over time. No cases of recurrent laxity or dimpling in the central neck were observed. The weight loss patients had stable weight one year before surgery and no further weight loss was pursued.

In the literature hypertrophic scarring after facelift surgery varies between $2-18 \%$ [22, 28, 29]. No hypertrophic scarring was found in this study due to tension free wound closure and a pure Caucasian patient collective.

In conclusion this retrospective study describes the simple reproducible complication free approach to restoring the neck after massive weight loss.

Our classification reflects deformities found in our patients.

With a stable weight it allows for accurate preoperative planning and a straightforward procedure that is possible to combine with other staged procedures in the weight loss patient. It results in a visible but pleasing scar as a tradeoff for improved neck contour of the weight loss patients. With 
consideration of the type of neck deformity and following the suggested treatment recommendations according to our grading scale in our hands this procedure is a great amendment to weight loss surgery.

\section{Conclusion}

Post-bariatric weight loss deformities are diverse, disordered, and often unpredictable. Our Classification of Neck Contour Deformities is structured and easily applied. Its standardized algorithmic approach for neck lift in massive weight loss patients may optimize the results without increasing the complication rate. Direct neck lift procedures prove to be reliable techniques with rare complication rates that can be performed together with other postbariatric procedures.

\section{Ethical Statement}

\section{Conflict of Interest}

The authors declare that they have no competing interests.

\section{Ethical Approval and Informed Consent}

We state that all procedures conformed to the Declaration of Helsinki. This study was approved by the appropriate Ethics Committee and informed written consent to publish personal and medical information was obtained from all patients.

\section{References}

[1] Cronin TD, Biggs T. The T-Z-plasty for the male "turkey gobbler" neck. Plast Recon Surg. 1971; 47 (6): 534-8.

[2] Biggs TM, Steely RL. The male neck and T-Z-plasty: 28 years later. Aesth Surg J. 2000; 20 (1): 31-4.

[3] Singer R. Improvement in the young fatty neck. Plast Recon Surg. 1984; 73 (4): 582-589.

[4] Gryskiewicz JM. Submental suction-assisted lipectomy without platysmaplasty. Plast Recon Surg. 2003; 112 (5): 1393-1405.

[5] Bitner JB, Friedman O, Farrior RT, Cook TA. Direct Submentoplasty for Neck Rejuvenation. Arch Facial Plast Surg. 2007; 9 (3): 194-200.

[6] Rohrich RJ, Rios JL, Smith PD, et al. Neck rejuvenation revisited. Plast Reconstr Surg. 2006; 118: 1251-63.

[7] Coon D, Michaels J 5th, Gusenoff JA, Purnell C, Friedman T, Rubin JP. Multiple procedures and staging in the massive weight loss population. Plast Reconstr Surg. 2010; 125 (2): 691-698.

[8] Giordano S, Victorzon M, Stormi T, Suominen E. Desire for body contouring surgery after bariatric surgery: do body mass index and weight loss matter? Aesthet Surg J. 2014; 34 (1): 96-105.

[9] Rubin JP, Jewell ML, Richter DF, Uebel CO. Body Contouring and Liposuction. Edinburgh, Scotland: Elsevier; 2012: 386.
[10] Klassen AF, Cano SJ, Scott A, Johnson J, Pusic AL. Satisfaction and quality-of-life issues in body contouring surgery patients: a qualitative study. Obes Surg. 2012; 22: $1527 \mathrm{e} 34$.

[11] Pelle-Ceravolo M, Angelini M, Silvi E. Treatment of Anterior Neck Aging without a Submental Approach: Lateral SkinPlatysma Displacement, a New and Proven Technique for Platysma Bands and Skin Laxity. Plast Recon Surg. 2017; 139 (2): 208-321.

[12] Song AY, Jean RD, Hurwitz DJ, Fernstrom MH, Scott JA, Rubin JP. A classification of contour deformities after bariatric weight loss: the Pittsburgh Rating Scale. Plastic Recon Surg. 2005; 116 (5): 1535-1544.

[13] Dindo D, Demartines N, Clavien PA. Classification of surgical complications: a new proposal with evaluation in a cohort of 6336 patients and results of a survey. Ann Surg. 2004; 240: 205-213.

[14] Don Parsa F, Castel N, Parsa NN. A modified, direct neck lift technique: the cervical wave-plasty. Arch Plast Surg. 2016; 43 : 181-188.

[15] Ziegler UE, Ziegler S, Zeplin PH. Allgemein- und Viszeralchirurgie up2date 2017; 11 (02): 149-172.

[16] Souther SG, Vistnes LM. Medial approximation of the platysma muscle in the treatment of neck deformities. Plast Recon Surg. 1981; 67 (5): 607-13.

[17] Feldman JJ. Corset platysmaplasty. Plast Recon Surg. 1990; 85 (3): 333-43.

[18] Zins JE, Fardo D. The 'anterior only' approach to neck rejuvenation: an alternative to face lift surgery. Plast Recon Surg. 2005; 115 (6): 1761-8.

[19] Yousif NJ, Matloub HS, Sanger JR. Hyoid suspendion neck lift. Plast Reconstr Surg. 2016; 138 (6): 1181-1190.

[20] Conway H. The surgical face lift-rhytidectomy. Plast Reconstr Surg. 1970; 45: 124-30.

[21] Kamer FM, Frankel AS. Isolated submentoplasty: a limited approach to the aging neck. Arch Otolaryngol Head Neck Surg. 1997; 123: 66-70.

[22] Friel MT, Shaw RE, Trovato MJ, et al. The measure of facelift patient satisfaction: the Owsley Facelift Satisfaction Survey with a long-term follow-up study. Plast Reconstr Surg. 2010; 126: 245-57.

[23] Larson JD, Tierney WS, Ozturk CN, Zins JE. Defining the Fat Compartments in the Neck: A Cadaver Study. Aesth Surg J. 2014, 34 (4): 499-506.

[24] Rohrich RJ, Pessa JE. The retaining system of the face: histologic evaluation of the septal boundaries of the subcutaneous fat compartments. Plast Reconstr Surg. 2008; 121: 1804-1809.

[25] Riefkohl R, Wolfe JA, Cox EB, et al. Association between cutaneous occlusive vascular disease, cigarette smoking, and skin slough after rhytidectomy. Plast Reconstr Surg. 1986; 77: 592-595.

[26] Pierpont YN, Dinh TP, Salas RE, Johnson EL, Wright TG, Robson MC, Payne WG. Obesity and surgical wound healing: a current review. ISRN Obes. 2014: 638936. 
[27] Parsa AA, Nakasone GK, Soon CW, et al. Smoking and breast reduction. Plast Reconstr Surg. 2006; 117: 2506-2507.

[28] Henderson J, O’Neill T, Logan A. Direct anterior neck skin excision for cervicomental laxity. Aesthetic Plast Surg. 2010; 34: 299-305.
[29] Matarasso A, Elkwood A, Rankin M, et al. National plastic surgery survey: face lift techniques and complications. Plast Reconstr Surg. 2000; 106: 1185-1195. 